\title{
Test de Clements
}

(Una prueba rápida para investigar el surfactante pulmonar en el líquido amniótico)

Dr. Marco Antonio Hernández $\mathrm{R}^{+}$

Instituto Materno Infantil 'Servicio de toxemias-Bogotá.

\section{INTRODUCCION:}

Como un recurso para conocer la concentración del surfactante en el líquido amniotico se describió el test de Clements, llamado también de la estabilidad de las burbujas, dependiente de la propiedad del material tensoactivo pulmonar en el líquido amniótico para generar burbujas estables enpresencia de una dilución específica de etanol. Es bien conocido que el test guarda paralelismo con la medida de la relación lecitina esfingomielina, así como la predicción del Sindrome de Dificultad respiratoria.

La relación L/E y el test de las burbujas no son solamente usados para el diagnóstico prenatal de maduración pulmonar puesto que también se usan para el diagnóstico post-natal del Sindrome de Dificultad Respiratoria. Blumenfeld y Borer (5.10.11), usan aspiración traqueal y faringea lo mismo que jugo gástrico para practicar el test y buscar la relación clínica de la enfermedad.

El surfactante pulmonar facilita una función físiológica que es absolutamente básica para la supervivencia extrauterina y la estabilidad alveolar. El surfactante se desarrolla y madura durante la vida fetal, y cuando la síntesis intrauterina se detiene, ocurre el síndrome de dificultad respiratoria, de alta mortalidad en el recién nacido según Gluck, L.(8.9).

El surfactante pulmonar es un compuesto que tapiza el alveolo del pulmón. Está compuesto por varias sustancias, incluyendo proteínas y carbohidratos, pero principalmente de líquidos. El principal componente es una lecitina altamente especializada de la

+Médico Residente III del departamento de Ginecología y Obstetricia de la Facultad de Medici.na, Universidad Nacional. superficie activa, que disminuye la tensión superficial y que contiene ácidos grasos saturados en ambos carbonos alfa y beta. La tensión superficial es vista diferentemente por biólogos y clínicos, lo mismo que por químicos y físicos. (Gluck 9.10). Fenómeno que ocurre en la unión aire líquido, lo mismo en un vaso de agua que en un alveolo. Es el resultado de las diferencias en las atracciones intermoleculares, de tal manera que el paquete más denso de moléculas en el líquido alveolar es más cerrado y está hacia abajo, en comparación con el grupo molecular más suelto, más disperso de la interfase aire de la parte superior. El resultado de esta atracción puede ser medida en. unidades de trabajo, o en el trabajo fuerza (Tensión superficial) entre la interfase aireagua que es de 72 dinas por centímetro (Gluck).

'Cuál es el significado clínico del surfactan: te? . La estabilidad pulmonar depende de él.

Los alveolos cambian su radio con la expi ración y la inspiración. En la inspiración el radio del alveolo aumenta; en expiración se hace considerablemente más pequeño. El efecto del cambio en el radio del alvéolo es mejor demostrado por el teorema de $\mathrm{La} \mathrm{Pla}^{-}$ ce que dice básicamente que la tensión de la pared de una esfera espansible es inversamente proporcional a su radio (Gluck.10). Los alveolos actúan como una bomba disminuyendo de tamaño con la expiración, la tensión de la pared aumenta marcadamente y si nó fuera por la sustancia estabilizadora, los alveolos disminuirían su tensión superficial y deberían colapsarse en expiración.

(Gluck.8.10).

La presencia del surfactante en Líquido Amniótico fue primero demostrado por Pattle en 1955, cuando mostró la estabilidad de las burbujas desde la superficie de corte del pulmón.

Shortly, Thereafter, Clements, (5.9), sobretodo este último, identificó fosfolípidos en el superfactante pulmonar, en particular lecitina. 
Dos enzimas son las responsables de la biosíntesis de lecitina en la producción del surfactante en los fetos humanos.

VIA I. TRANSFERAZA DE FOSFOCOLINA (citidilo)

Ac. Palmítico - O $\begin{array}{r}\text { O- Ac. Palmítico } \\ \text { P04-CH2CH2NH }\end{array} \underset{\mathrm{CH} 3}{\mathrm{CH} 3}$

P04 - de citidina

Fosfato de colina +diglicérido Lecitina de dipalitoilo

Madura a las 35 semanas de gestación en el hombre.

VIA II. METIL TRANSFERAZA

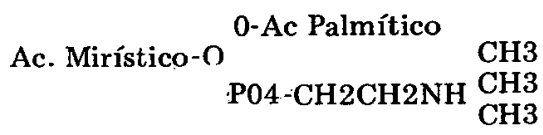

Etanolamina de fosfatidilo $+\mathrm{CH} 3$ (de metionina-S-adenosilo-L)

Metionina Lecitina de palmitoilo miristoilo.

Este patrón es identificable tempranamente, a las 22-24- semanas; la actividad de esta enzima aumenta durante la gestación y es más activa a término. (Gluck 9.13).

\section{PREMATUREZ-DESARROLLO DEL PUL-} MON FETAL Y S. D. R.

El sindrome de dificultad respiratoria (S.D. R.) es un complejo sintomático, que afecta principalmente a los lactantes prematuros. Según Cohen Dunn y Gregg (4.6.17) niños a término por la fecha o por el peso al nacimiento, que desarrollan síndrome clásico de dificultad respiratoria șon aquellos en quienes los pulmones no están maduros al nacimiento; siendo esta falta de madurez pulmonar, independiente de la edad de gestación o del peso al nacimiento.

En los lactantes nacidos de madres diabéticas o después de operación cesárea cưando el pulmón no está todavía maduro desde el punto de vista bioquímico, puede presentarse el síndrome de dificultad respiratoria, porque nacen demasiado pronto, bien sea por inducción o por la misma cesárea. Sin embargo, no parece existir nada específico en relación con la diabetes materna o la in- tervención cesárea en que predisponga al recién nacido a padecer sindrome de dificultad respiratoria, porque nacen demasiado pronto, bien sea por inducción o por la misma cesárea. Sin embargo, no parece existir nada específico en relación con la diabetes materna o la intervención cesárea en que predisponga al recién nacido a padecer síndrome de dificultad respiratoria; siendo quizá el común denominador determinante la inmadurez bioquímica de los pulmones. (18.19.28).

Se ha dicho que las fuerzas ejercidas sobre el tórax durante el trabajo de parto ayuda a eliminar el líquido de los pulmones más facilmente de lo que ocurre en lactantes nacidos por cesárea. Sin embargo, no es probable que el trabajo de parto ejerza tal influencia sobre la maduración del pulmón o sobre la maduración del pulmón o sobre el curso del síndrome de dificultad respiratoria.

El pulmón fetal es un órgano metabolicamente activo que segrega líquido en la cavidad amniótica. Carmal y cols(3)ligaron la traquea de fetos de conejos y observaron distensión subsiguiente del pulmón.

Adams y otros (1.17) han informado que el líquido traqueal es más ácido ( $\mathrm{Ph} 6.4 \mathrm{com}-$ parado con 7.07) y que contiene menos bicarbonato que el líquido amniótico y demostraron que el líquido pulmonar difiere en osmolalidad total y en concentración de proteínas, úrea y bicarbonato del líquido amniótico. El volumen del líquido en el en el pulmón se ha estimado en $40-80 \mathrm{~m}$ l $(10-20 \mathrm{mlx} \mathrm{Kg})(28)$.

La aireación del pulmón al nacimiento no es la insuflación de un órgano vacio y colapsado, sino la sustitución rápida del líquido intraalveolar por aire(29).

Se ha comprobado que algunas entidades maternas y fetales se acompañan de retrazo en la maduración del pulmón del feto tales como diabetes sacarina, glomerulonefritis y algunos casos de hidropesia fetal (3.6)

\section{CAUSA DEL SINDROME DE DIFICUL- TAD RESPIRATORIA}

La síntesis de lecitina tensoactiva por la via de metilación permite al feto sobrevivir como prematuro una vez nacido. Esta vía es muy sensible a los cambios de $\mathrm{Ph}$ por lo cual, con la acidosis propia del sindrome, disminuye la actividad de la enzima de la 
transmetilación $2(20.21 .22)$.

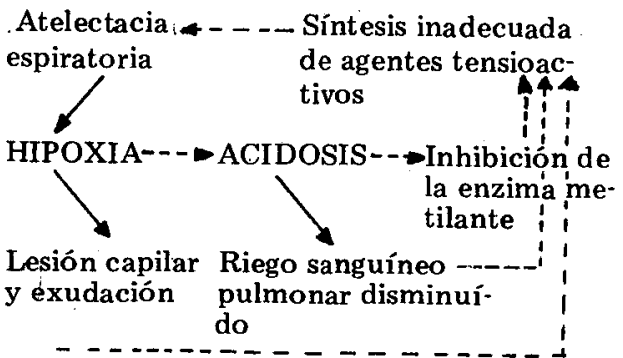

Desde la primera respiración, al poco tiempo de haber nacido el niño que desarrolla el sindrome de Dificultad Respiratoria, comienza a tener taquipnea y respiraciones dificultosas, desarrolla un quejido audible; las respiraciones continúan más laboriosas y comienza el uso de los musculos accesorios de la respiración. La radiografía de tórax muestra un difuso patrón retículo granular con un broncograma aéreo.

Los cambios patológicos de atelectasia progresiva y el edema pulmonar son progresivos y secundarios a todos los procesos des-

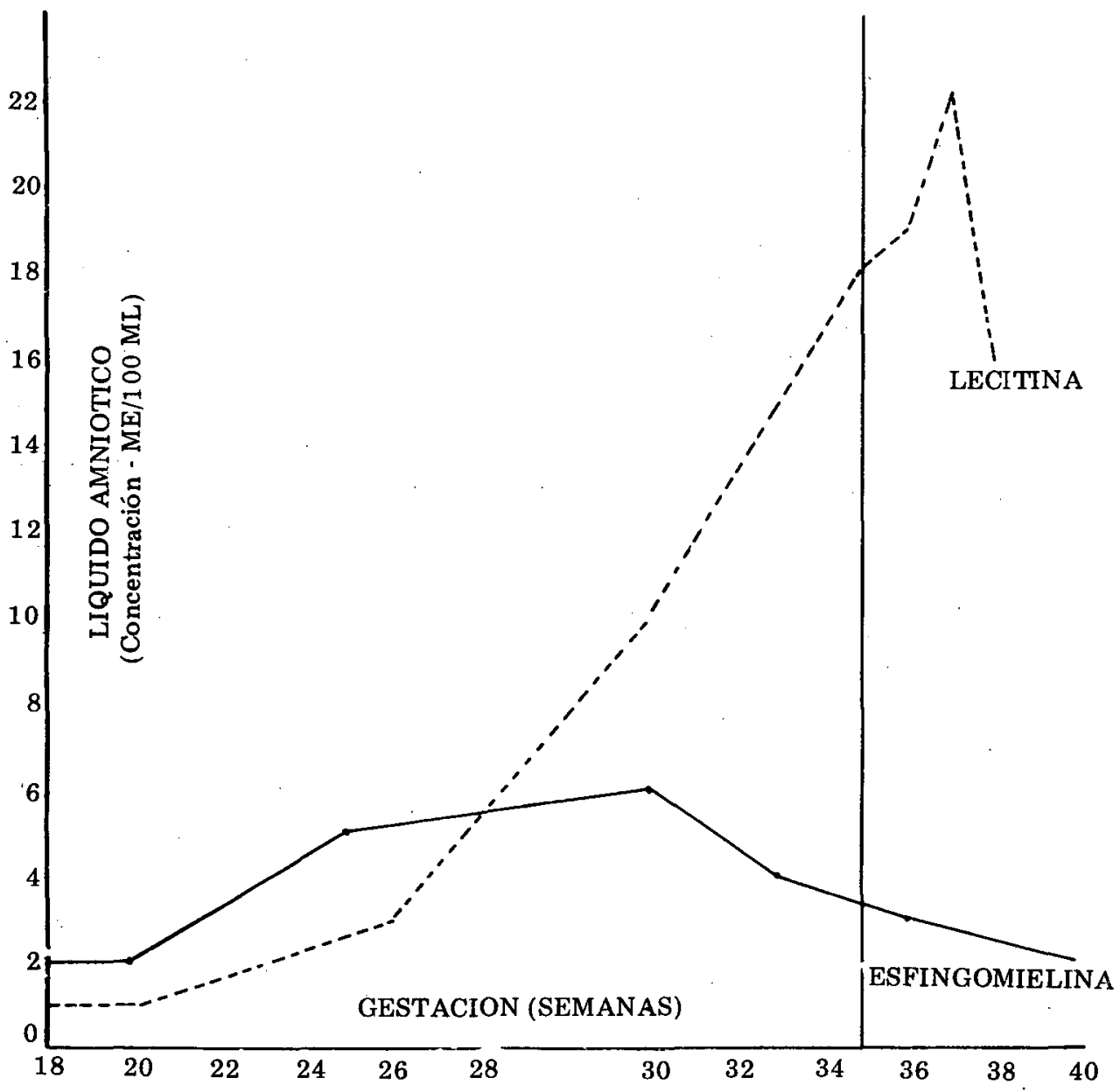

Gráfica No. 1. Relación lecitina esfingomielina en las difereptes semanas de embaràzo. 
critos; con el colapso pulmonar hay hipoxia que lleva a la acidosis, dismínuye el riego sanguíneo pulmonar, disminuye la producción de surfactante y se promueve el colapso. La hipoxia también daña el endotelio capilar que por la alta presión intratoráxúca. negativa ayuda a producir la transudación del fluído dentro del alvéolo(Gluck)(8.9.10. 11.12)

Se acepta en la actualidad, como método para determinar la madurez pulmonar en forma cuantitativa el examen del líquido el lapso de los últimos 5 años.

Se practicó amniocentensis apacientes preeclánticas y eclámpticas extrayéndose en promedio $10 \mathrm{cc}$. de líquido amniótico para para la prueba; se observó el aspecto del líquido y de su contenido. Las punciones traumáticas o contaminadas se desecharon.

El sitio de la punción se escogió de acuerdo a la facilidad técnica y se siguieron los pasos que aparecen en las fotografías. En ninguna de las punciones hubo complicaciones

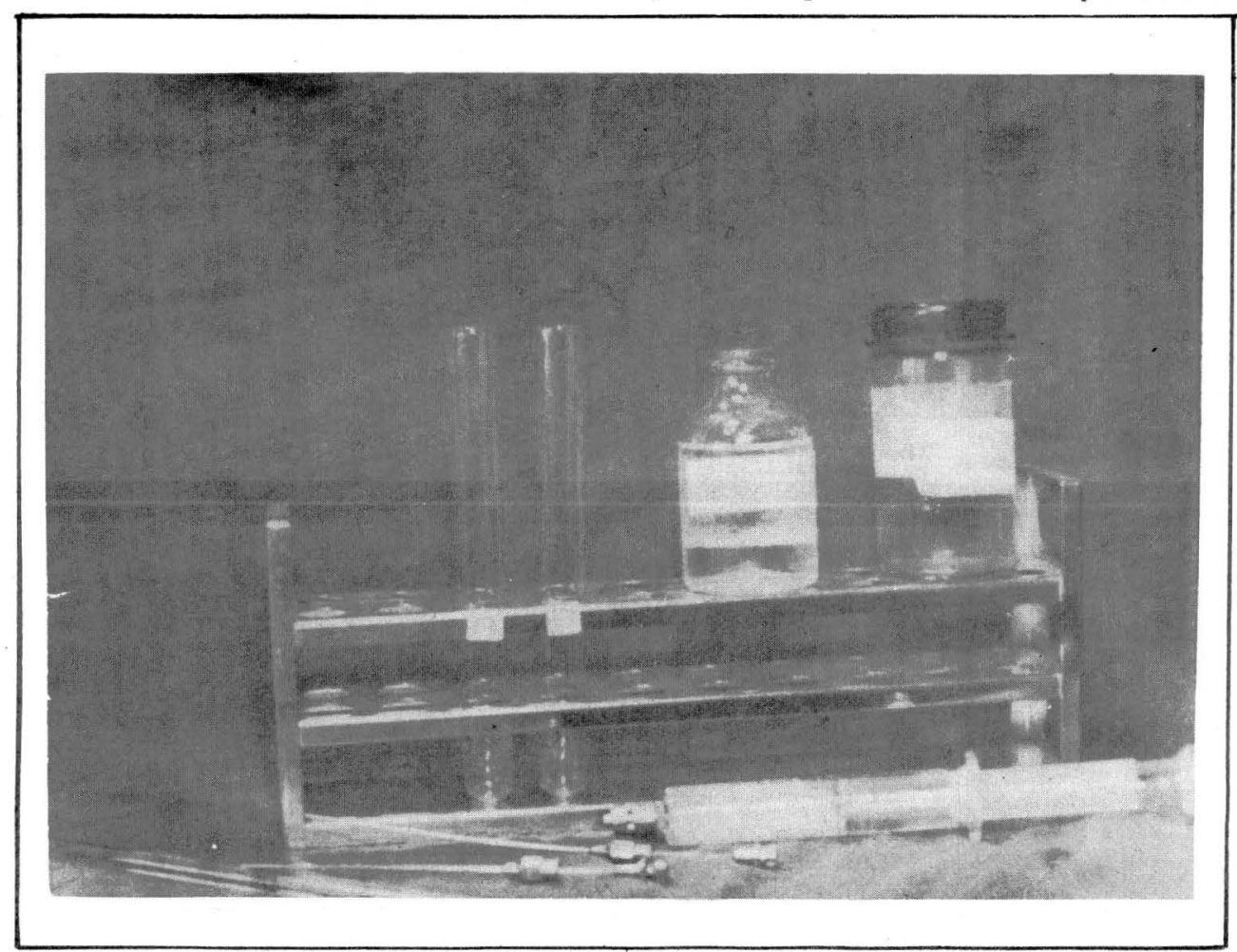

amniotico en busca de fosfolípidos, sabiendo que después de la semana 35 se encuentra una relación entre lecitina-esfingomielina (L-E) de 2:1 que proporciona una madurez suficiente para la vida extrauterina. (Pattle y cols. 25-27-26).

\section{MATERIAL Y METODOS}

El presente trabajo se realizó en el servicio de Toxemias del Instituto Materno Infantil "Concepción Villaveces de Acosta:: con una muestra de 100 pacientes, hospitalizadas en graves para la madre ni para el feto.

Una vez recolectada la muestra del líquido amniótico, se procedió a practicar el test ideado por Clements, basado en la habilidad del surfactante pulmonar para generar burbujas estables en presencia de etanol. El analisís del trabajo de Clements con $138 \mathrm{ca}^{-}$ sos demostró que el surfactante aparece al rededor de la semana 33 , aunque el tiempo. de aparición es variable desde la semana 25 hasta el término y se clasifica en tres tipos: claramente negativo, intermedio o dudoso 


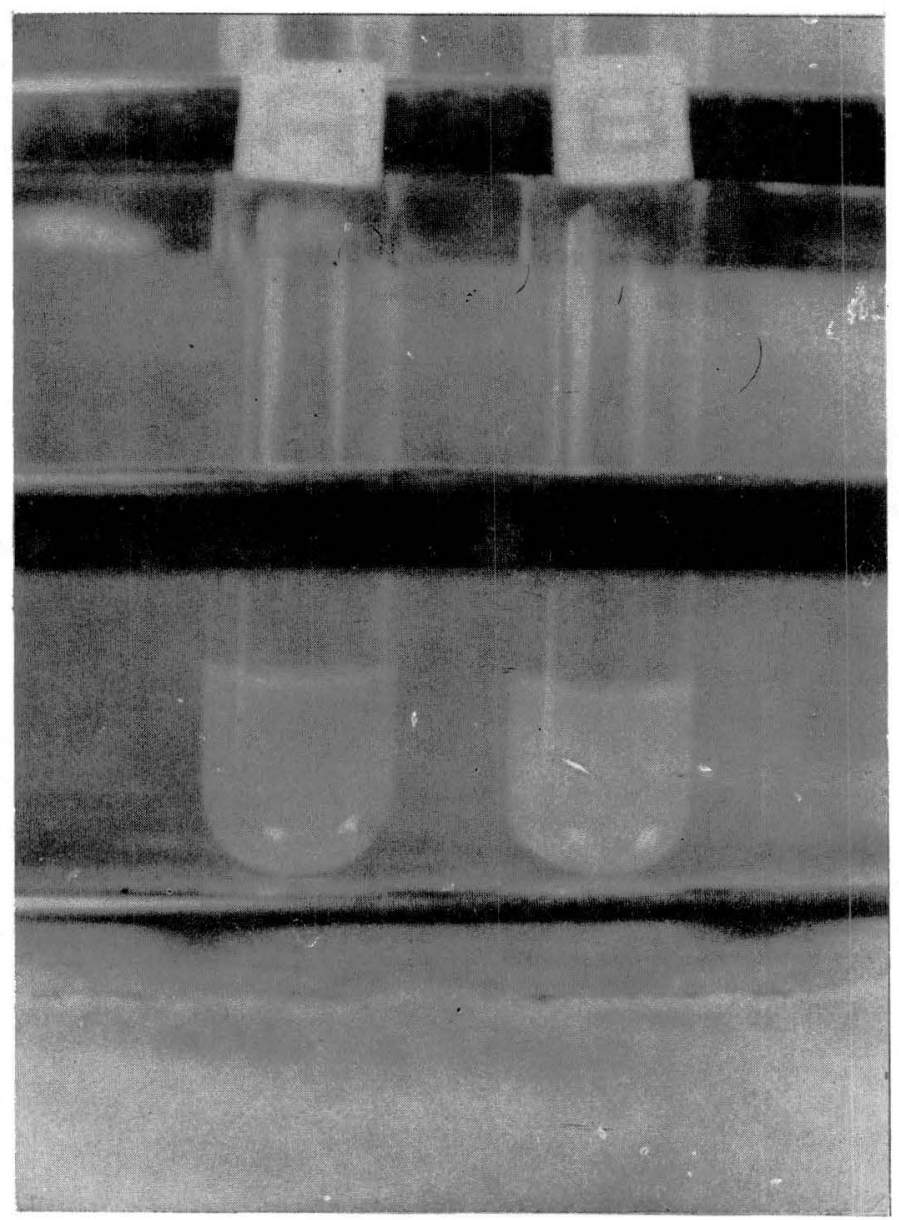

TEST NEGATIVO

y claramente positivo (Clements 5 ).

Algunas otras sustancias del líquido amniótico tales como proteínas, sales biliares, ácidos grasos libres; pueden también formar burbujas estables, pero estas son excluídas de la película estable con el etanol. (Clements, Gluck 5.1.)

Si es posible se realiza el test inmediatamente, sino, se puede guardar durante una hora en refrigerador a 5 grados centígrados.
Cuando el líquido no puede ser analizado en el mismo día, es congelado a -20 grados. Inmediatamente antes del análisis el tubo que contiene el líquido es invertido suavemente varias veces para obtener una suspensión uniforme de partículas sin provocar la formación de burbujas.

Se colocan 5 tubos de vidrio lavados con agua pura y secos y se añaden $1,0.75,0.50$, 0.25 y 0.20 mls. de líquido amniótico res- 


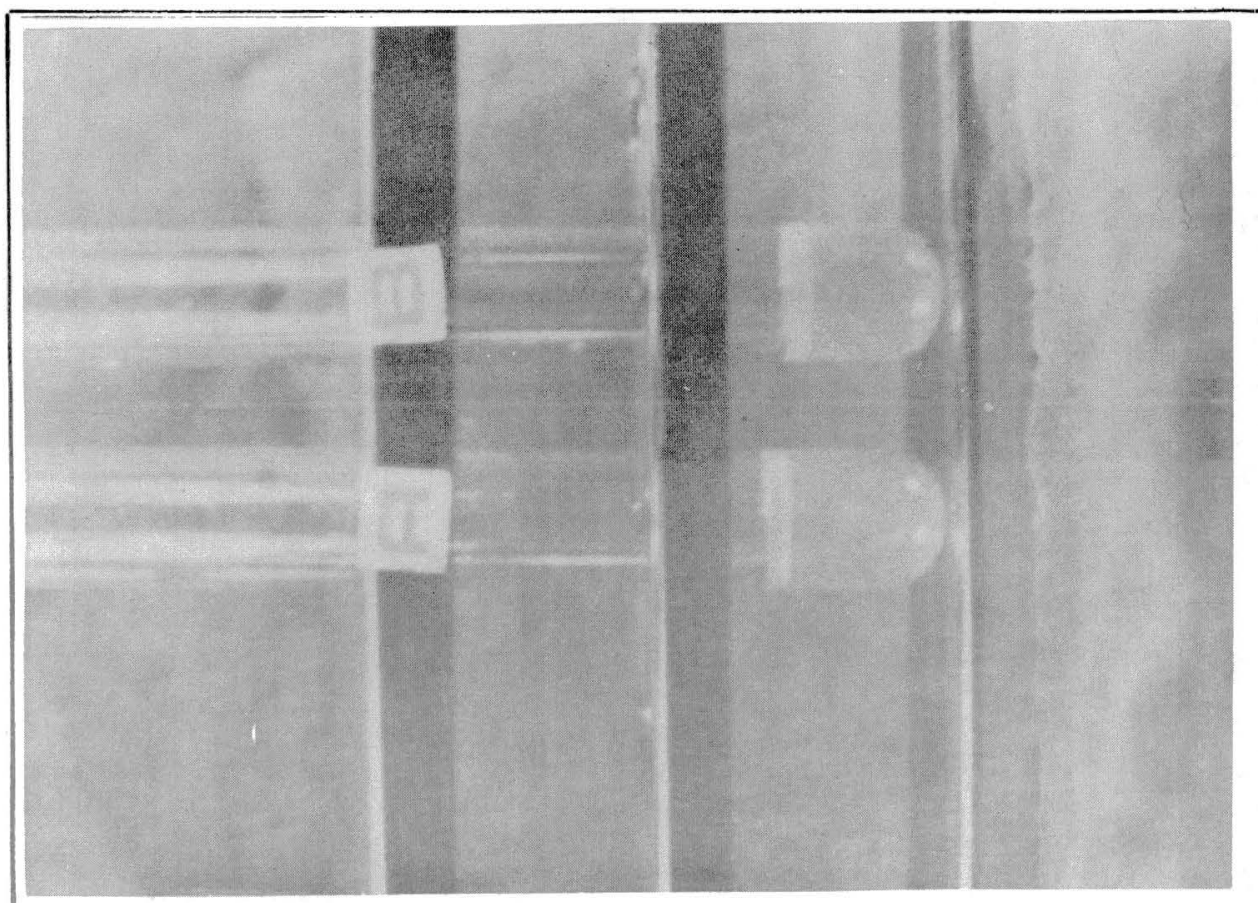

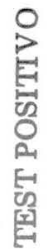

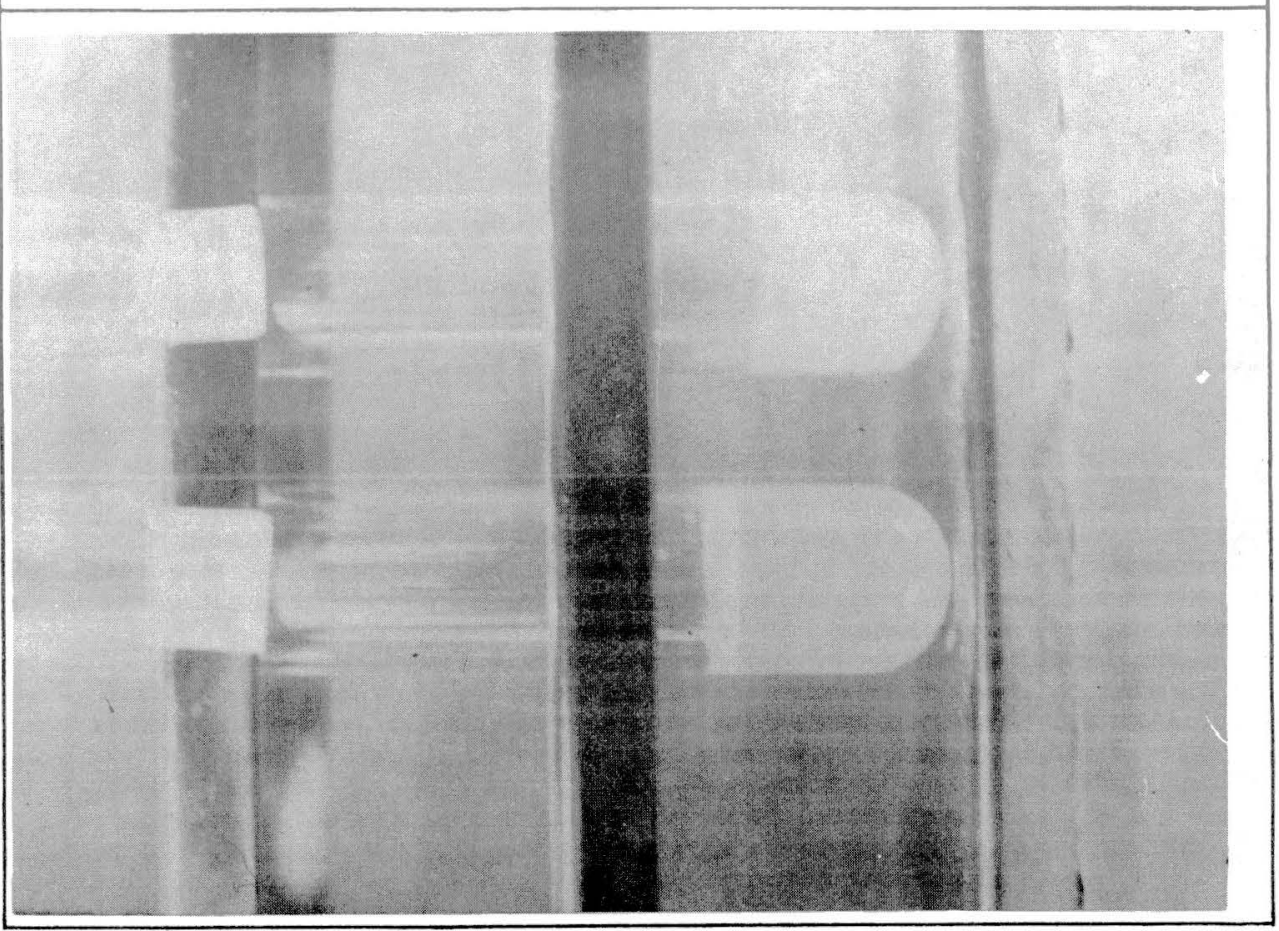

0
क
0
0
0
0
-1
0
019
[- 


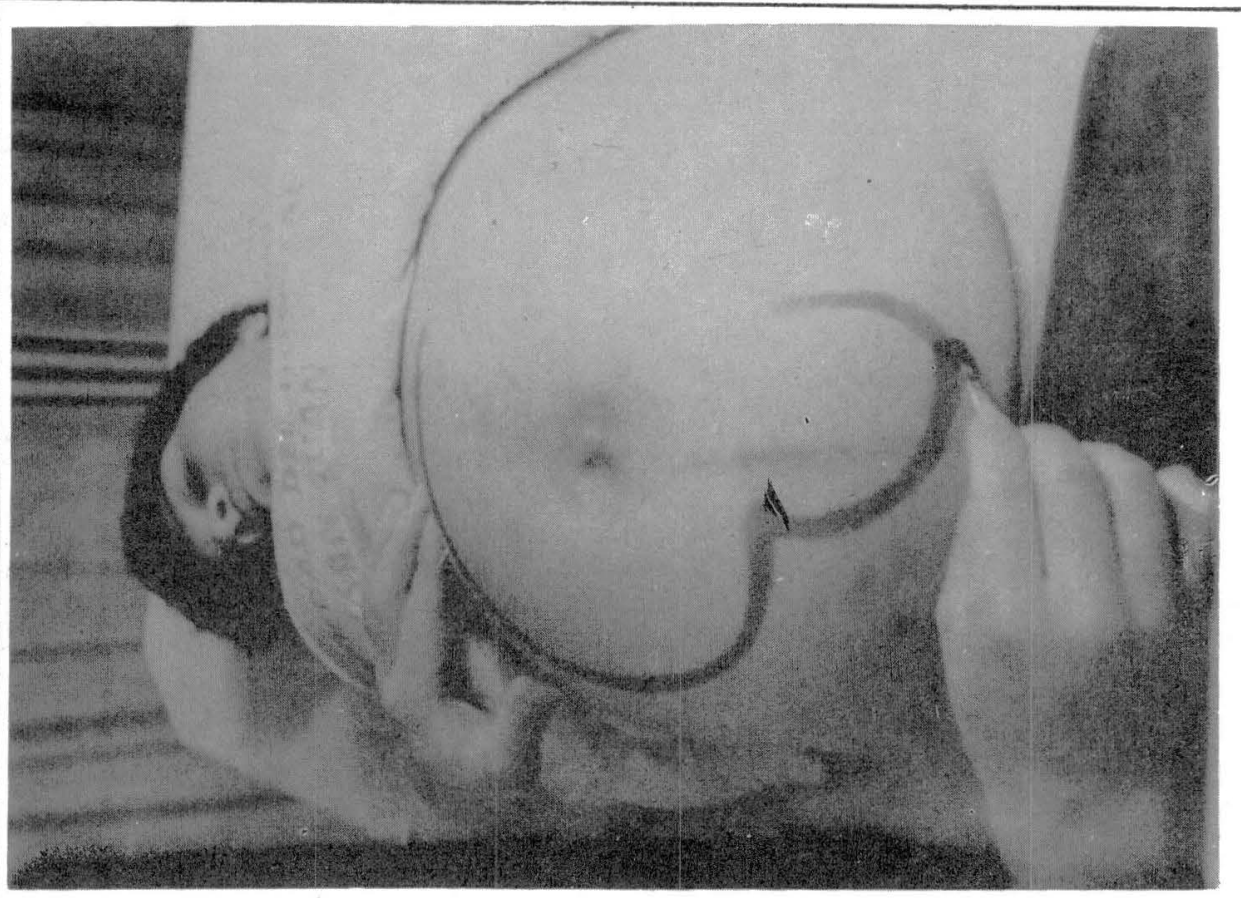

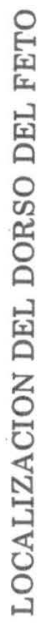

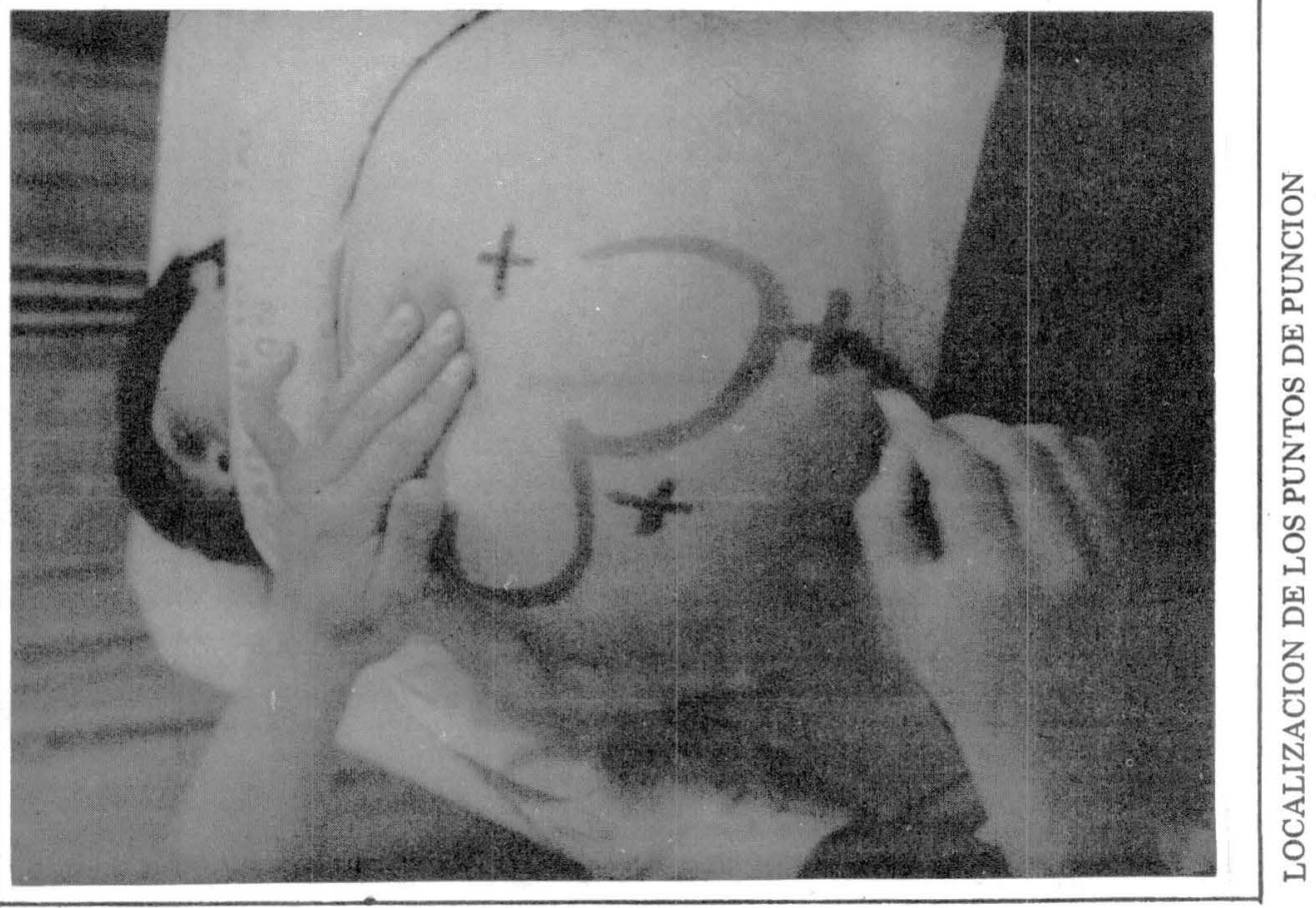




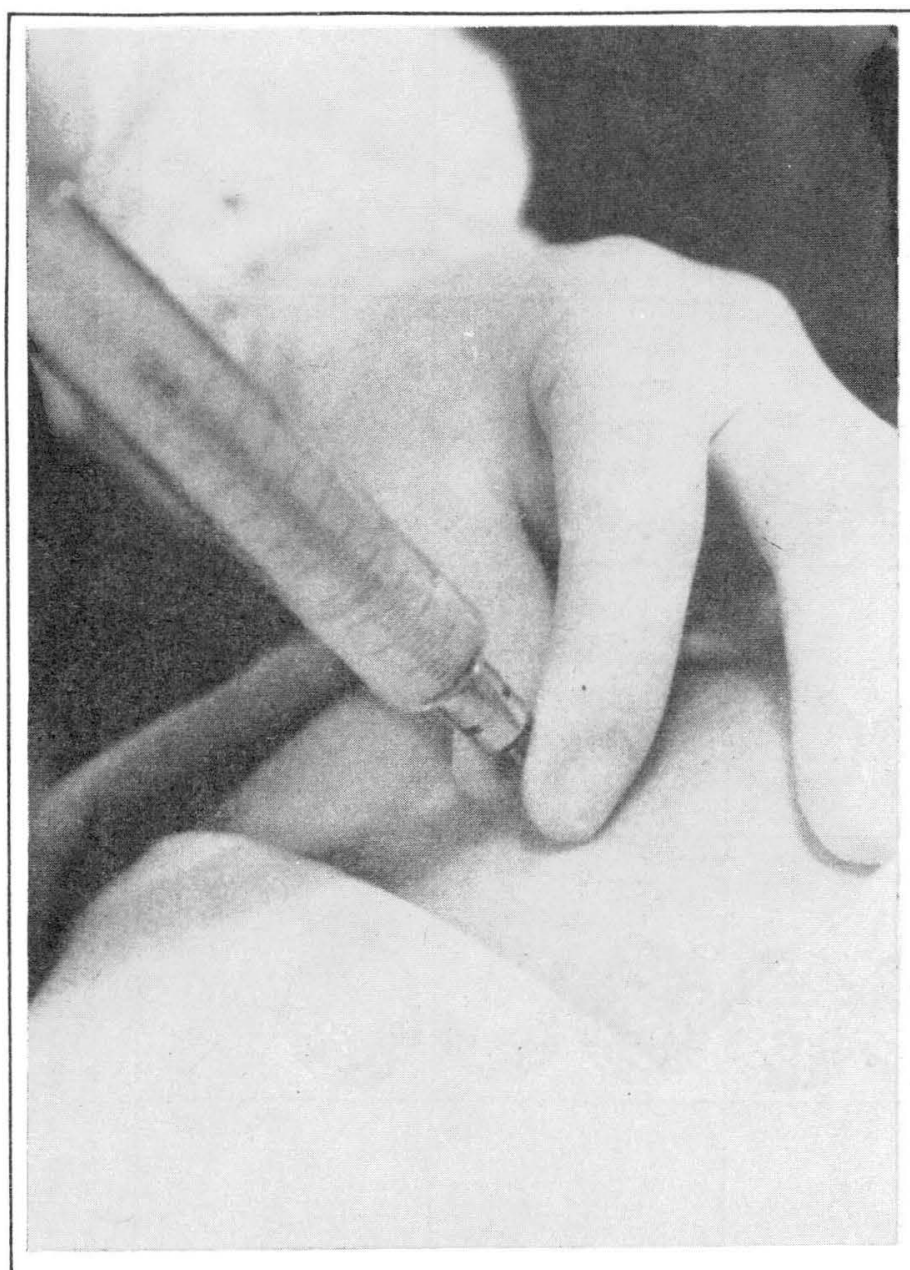


pectivamente; luego $0.25,0.50,0.75$ y 0.80 mls. de solución salina al $0.9 \%$, con pipeta en los tubos $2,3,4,5$ respectivamente; por último un ml. de etanol al $95 \%$ se añade a cada tubo; los tubos se tapan y se agitan vigorosamente durante 15 segundos y luego se colocan verticalmente en una gradilla; 15 minutos después aparece la interfase airelíauido la cual es examinada; se considera positiva si se han formado pequeñas burbujas estables en toda la cirćunferencia deI menisco. En el presente trabajo se usaron solamente 2 tubos, utilizando diluciones al $1 \mathrm{y}$ al $1 / 2$.

Los tubos de vidrio deben ser claros, sin remanentes de jabón, suero o líquidos biológicos que pueden producir burbujas. Se usan tubos de un diámetro interno de 14-18 milímetros. El diámetro del tubo podría afectar la estabilidad de la burbuja por los cambios en el área de la superficie del vidrio en contacto con la burbuja. En los tubos de mayor diámetro es más difícil observar las burbujas finas. Una vez que se produce la burbuja, es conveniente no mover los tu- bos. No se debería hacer el test cuando está el líquido teñido por meconio puesto que el meconio dá un test positivo cuando se presentan concentraciones bajas; euando contiene sangre sucede lo mismo, en cuyo caso debe hacerse el test después de contrifugar por 5 minutos a 5 revoluciones por minuto para separar los glóbulos rojos.

La centrifugación de las muestras a altas revoluciones por largos periodos no debe hacerse porque precipita el surfactante y cuando se hace el "test" con el líquido sobrenadante es negativo. El Iíquido deberá ser extraído directamente de la cavidad amniótica para evitar contaminación; tampoco se hará cuando haya duda de si la muestra es o nó líquido amniótico.

El líquido ascítico del feto in útero y del recién nacido, han dado "test" positivo. Como la sangre y el meconio, el líquido ascítico contiene suficiente cantidad de lecitina saturada y esfingomielina para estabilizar una cantidad visible de burbuias en nresencia de $47.5 \%$ de etanol. (Clements, Adams y-cols. 2.5.25.30.31.)

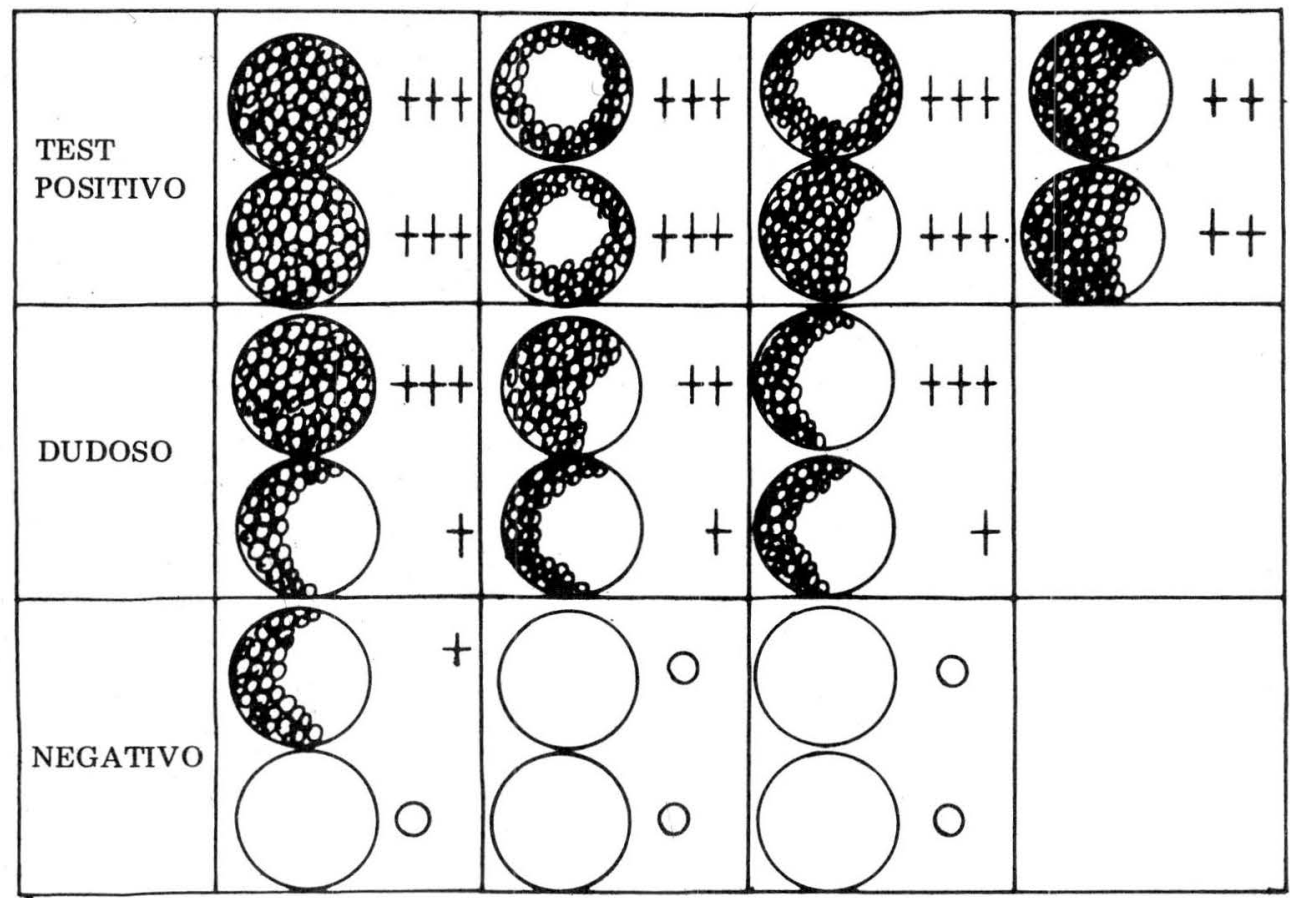

Gráfica No. 2. Esquema de las burbujas en los tubos mostrando los resultados positivos dudosos o negativos. 
Para poder determinar el futuro del feto y poder predecir más o menos con alguna exactitud si el niño va a sufrir la enfermedad de membrana hialina, se clasifican las pruebas en positivas, dudosas y negativas según se ilustra en el siguiente esquema.
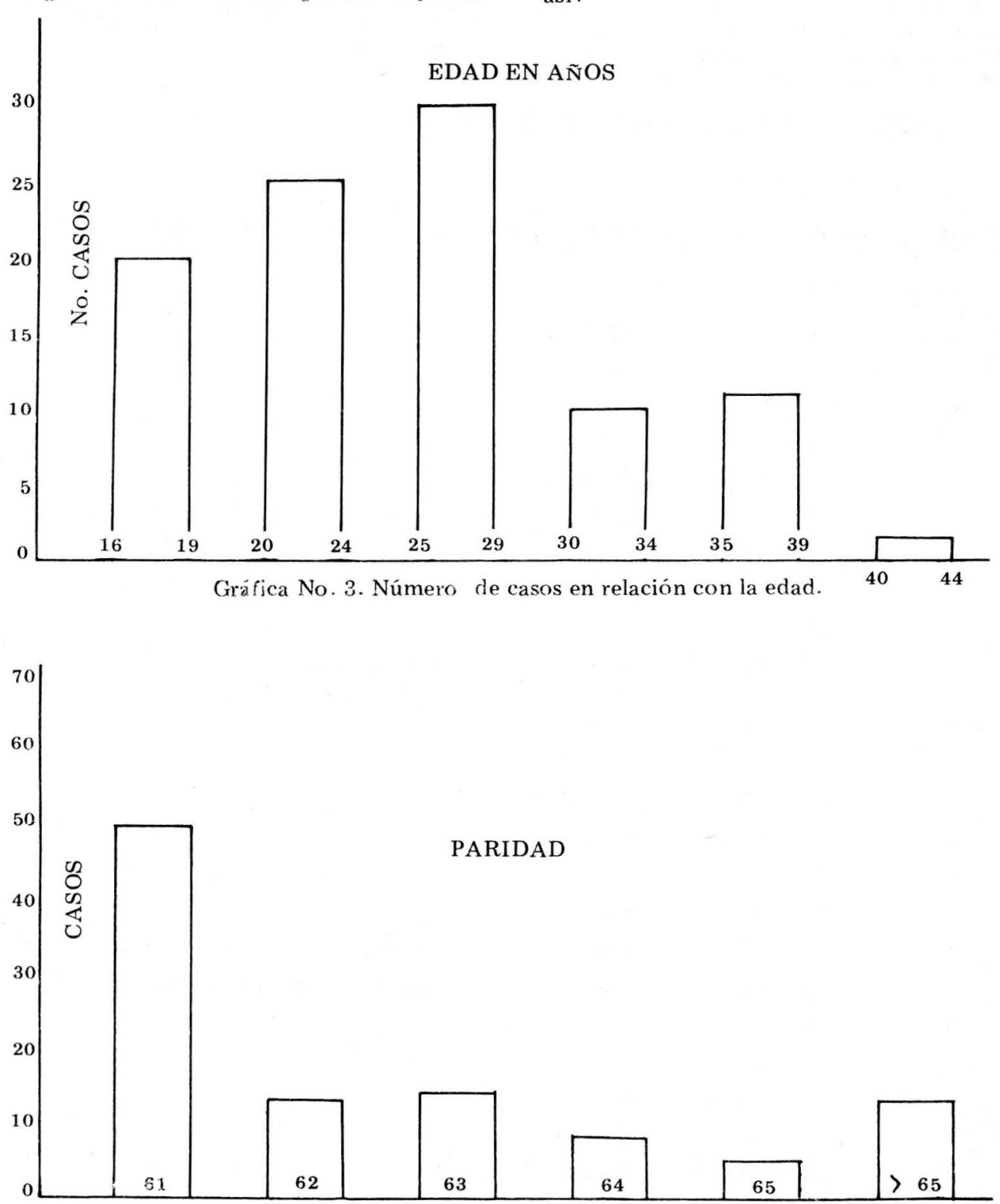

(Gluck, Monserrat. 16.24).GRAFICA no. 2 RESULTADOS

Se practicó amniocentesis a 100 pacientes; que se clasificaron según su edad y paridad, así: 
Como se puede ver claramente en la gráfica el mayor grupo de pacientes estudiadas estuvo compuesto por muieres entre 25 y 29 años siguiendo en orden de frecuencia el grupo de 20 a 24 años, que junto con las de 16 a 19 años correspondió a pacientes que consultaron por toxemia pura, en la mavoría de los casos, a diferencia de los grupos de enfermas por encima de 35 años que llegaron al hospital, en su mayor parte con problemas hipertensivos crónicos agudizados por toxemia sobreagregada.

GRAFICA no. 4

El mayor número de muestras se obtuvieron de pacientes primigestantes, número este que fue disminuyendo con la paridad para volver a tener un ascenso en pacientes con más de cinco gestaciones.

\section{RESULTADO DEL “TEST” EN 27 PACIENTES ECLAMPTICAS}

EDAD EMBARAZO

\section{TEST Prueba Parto RECIEN NACIDO OBSERVACIONES}

\begin{tabular}{|c|c|c|c|c|}
\hline+ & 33 & 34 & Normal & \\
\hline+ & 34 & 36 & Normal & \\
\hline+ & 38 & 38 & Normal & \\
\hline+ & 34 & 34 & Normal & \\
\hline+ & 35 & 35 & Normal & \\
\hline+ & 36 & 37 & Normal & \\
\hline+- & 34 & 34 & Murió & Prematurez, Membrana Hialina \\
\hline+- & 34 & 33 & Murió & Prematurez, Membrana Hialina \\
\hline+- & 33 & 34 & Murió & Prematurez, Membrana Hialina \\
\hline+ & 34 & 34 & Normal & \\
\hline+ & 34 & 34 & Murió & $\begin{array}{l}\text { Sepsis, Bronconeumonía } \\
\text { Malformaciones congénitas }\end{array}$ \\
\hline+ & 35 & 35 & Normal & \\
\hline+ & 34 & 36 & Normal & \\
\hline+ & 35 & 39 & Normal & \\
\hline+ & 36 & 36 & Normal & \\
\hline+ & 36 & 36 & Normal & \\
\hline+ & 38 & 38 & Normal & \\
\hline+ & 36 & 36 & Murió & Prematurez, Membrana Hialina \\
\hline+- & 30 & 30 & Murió & Prematurez, Membrana Hialina \\
\hline+- & 39 & 39 & Normal & \\
\hline+ & 36 & 36 & Normal & \\
\hline+ & 37 & 38 & Normal & \\
\hline+ & 38 & 38 & Normal & \\
\hline+ & 36 & 37 & Normal & \\
\hline+ & 37 & 38 & Normal & \\
\hline- & 30 & 30 & Murió & Prematurez, Membrana Hialina \\
\hline
\end{tabular}


De los 100 casos estudiados, 27 correspondieron a pacientes eclampticas a las cuales se les practicó el "test" de Clements; 21 dieron resultados positivos; 5 casos dudosos y 1 negativo. Seis casos correspondieron a recién nacidos que fallecieron por membrana hialina. GRAFICA No. 5 y los casos dudosos en los cuales la positividad no fue franca se presentaron con mayor frecuencia en la semana 34 . De los 100 casos estudiados 52 resultaron positivos, 25 dudosos y 23 negativos.

Al $78.84 \%$ de los casos positivos, es decir

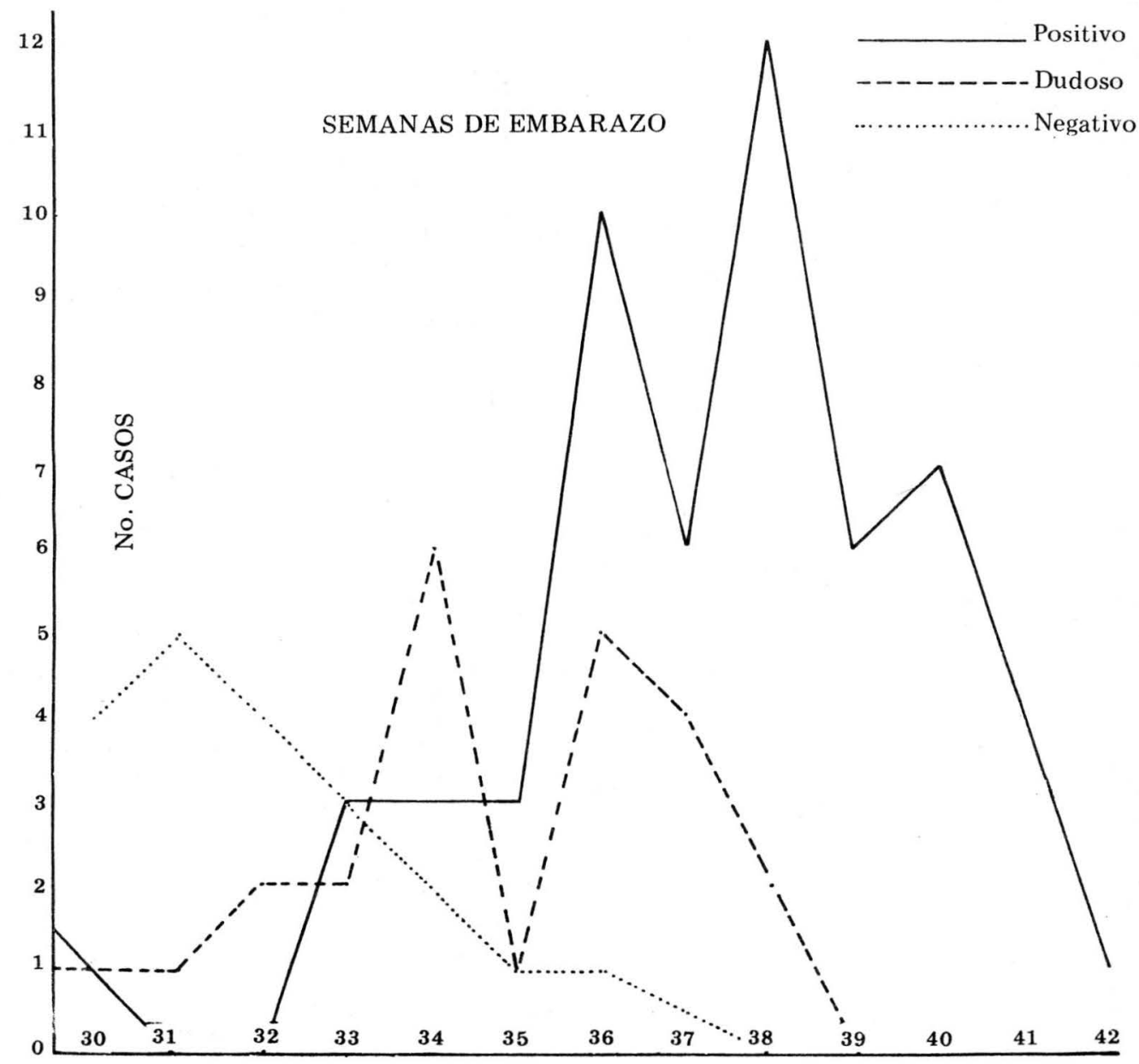

Gráfica No. 5. Edad del embarazo y su relación con el resultado del Test.

La gráfica nos demuestra claramente que el mayor número de casos positivos para burbujas estables, se presentó en edades avanzadas del embarazo, con el máximo de resultados positivos hacia la semana 38 . Por el contrario los casos negativos se presentaron con su máximo pico en la semana 31 ;
41 pacientes, se les practicó el 'test' entre las semanas 36-42 con resultados favorables para los recién nacidos.

Al $21.16 \%$ restante de los casos positivos que corresponde a 11 pacientes, se les practicó el "test" entre las semanas $30-35$, de estos 4 recién nacidos murieron tres por 
Membrana Hialina y el otro por Sepsis y $\mathrm{B} /$ neumonía todos a las 34 semanas de gestación.

Los otros 7 restantes cón "test" positivos, a quienes se les practicó la prueba alrededor de la semana 35 nacieron después de la semana 36 y fueron normales.

De los 25 casos dudosos el $64 \%$ o sea 16 muestras se efectuaron entre las semanas 36 a 38 con productos normales; el $36 \%$ restante correspondió a 9 muestras realizadas entre las 33 y 34 semanas de gestación murieron 5 recién nacidos por Membrana Hialina y 4 por Anoxia Intrauterina.

Por último, se presentaron 23 casos negativos de los cuales 16 pacientes tuvieron partos normales y recién nacidos sanos por lograr tener sus partos en edades más avanzadas del embarazo cuando indudablemente sus productos ya eran maduros. De los 7 recién nacidos que murieron 4 fueron por membrana hialina a las $30-31-33$ y 34 semanas respectivamente, los otros tres casos por causas diferentes como isoinmunización y anoxia intrauterina.

\section{RESUMEN Y CONCLUSIONES}

El primer riesgo que debe afrontar el recién nacido prematuro es el ajuste satisfactorio de su respiración y la primera "enfermedad" que encara es el complejo fisiopatológico descrito como el Sindrome de Dificultad Respiratoria. De su conocimiento y manejo adecuado depende en buena parte la posibilidad de reducir la morbimortalidad perinatal.

Puesto que la toxemia grávida y las enfermedades afines facilitan la frecuente ocurrencia del parto prematuro, o se hace imperiosa su provocación, es conveniente analizar concienzudamente tal eventualidad y sus implicaciones desde el punto de vista fetal. La posibilidad de contar con los recursos que brinda la amniocentesis hace que el procedimiento no deba omitirse.

Al lado de los exámenes más elaborados y sutiles, el "test" de Clements, correctamente efectuado, es un procedimiento sencillo, rápido y que muestra, de manera muy aproximada, el grado de maduración fetal.

Puesto que el empleo de los corticoides parece haber demostrado acción inductora de la formación del surfactante pulmonar, los estudios al respecto deben incrementarse. Su empleo en pacientes con toxemia grávi- da, en la cual existe un compromiso suprarenal no solo materno sino también fetal, debe ser analizado puesto que su administración a tales enfermos puede, eventualmente, resultar contraindicado. En contraste con lo anterior el empleo con ese fìn de. los barbitúricos, parece por nuestra experiencia, haber aprobado su bondad. En cuanto a la disminución de dificultad respiratoria por membrana hialina se presentan los resultados obtenidos en el "test" de Clements practicado a 100 pacientes atendidas en el "SERVICIO DE TOXEMIAS" del Instituto Materno Infantil de Bogotá.

Se tomaron muestras de líquido amniótico en diferentes semanas de gestación y se demostró que los recién nacidos antes de la semana 34 casi siempre tuvieron "test" negativos o dudosos y sufrieron el Sindrome de Membrana Hialina en contraste con los nacidos a partir de la semana 35 que, con algunas excepciones, dieron pruebas positivas y no padecieron la enfermedad.

\section{SUMMARY}

The first risk that a premature newborn must face is the satisfactory adjustment of his breathing and the first "illness' is the physiopatological complex described as a respiratory difficulty syndrome. The possibility of reducing perinatal mortality depends to a great extent on the identification and treatment of this syndrome.

Since serious Toxemia and similar diseases are a frecuent cause of premature births, or the necessity to induce them, it is advisable to carefully analize this issue and its implications from the fetal point of view. The possibility of making use of the resources made available by amniocentesis makes this procedure one that we cannot fail to include.

In comparison with the most elaborate and delicate exams, the Clements Test, when carried out effectively is a simple and quick procedure which shows quite accurately, the degree of fetal maturity.

Since the use of corticoides has apparently resulted in being an inductor agent in the formation of lung surfactant, studies should be intensified. Their use in patients with serious Toxemia with the presence of suprarenal complications, both maternal and fetal, should be analized since their administration to such patients may eventually prove to be unadvisable. In contrast with the 
aforegoing, the use of barbiturates for this purpose, with our past. experience, has shown its effectiveness.

In so far as a decrease in respiratory difficulty of the Hyaline membrane is concerned, the results obtained with the Clements Test on 100 patients attended at the "TOXEMIA SERVICE' section of Bogota's Maternal-Infant Institute, are presented. Samples of the amniotic fluid were taken at different stages of gestation, and it was revealed that those babies born before 34 weeks almost invariably suffered from $\mathrm{Hya}^{-}$ line Membrane Syndrome and had negative or undefined results on the "Test", in comparison with those born after 35 weeks who with few exceptions, had positive results and did not suffer from the desease.

\section{BIBLIOGRAFI A}

1. ADAMS, F.H. Fujiwara T. y Rowshan G.: J. Pediat, $51: 881,1.963$

2. ADAMS, F.H. Latta, H. El Salawy, A. y cols.: J. Pediat, 75:59, 1.969

3. CARMEL, J.A, Friedman, F y Adams, F.H.: Amer, J. Dischil 109:452, 1.965

4. COHEN, M.M. Weantranb, D.H. y Lilienfield, A.M.: Pediatrics 26:42. 1.960

5. ClementS, J.A. M.D. Arnold C.G. Platzer, M.D. Assessment of the risk of the respiratorydistress syndrome by a rapid test for surfactanin amniotic fluid. The New England Journal of Medicine, 18:286: 1.972

6. DUNN, P.M.: Arch. Dis Child, 40:62, 1.965

7. FUJIWARA, T., F.H. y Scudder, A.: J. Pediat. $65: 824,1964$

8. GLUCK, L.: Clin. Pediat de Norteamérica. 19: $325,1.972$

9. GLUCK, L. Kulovich, M.V. Eidelman, A.J. Pediat, Res $6: 81,1.972$

10. GLUCK, L.: Pediatrics, 49:465, 1.972

11. GLUCK, L.: Kulovich, M. Bose, R.C. y col.:
Amer. J. Obstetric Ginec, 109:440, 1.971

12. GLUCK, L.: Surfactant: 1.972: Clin. Pediat. de Norteamérica 19:325, 1.972

13. GLUCK, L.: Sribney, M. y Kulovich, M.V.: Pediat. Res. 1:247, 1.967

14. GLUCK, L.: Landowne,, R. A. y Kulovich, M. V.: Pediat. Res, 4:352, 1.970

15. GLUCK, L. Kulovich, M.V., Fdilman, A.J. y col.: Pediatric. Res, 6:81, 1.972

16. GLUCK, L. y Kulovich, M.V.: Amer. J. Obstetric, Gynec, 115:539, 1.973

17. GREGG, R.H. y Bernstein, J.: Amer. J. Dis. Child, 102:871 1.961

18. GEllis, S.S. y Hsia, D.Y.Y.: Amer. J. Dis. Child, 97:1, 1.959

19. HESS. O.W.: Amer. J. Obstet. Gynec, 75:376, 1.958

20. HEINEMANN, H.O. y Fishman, A. P.: Physiol 24: 3 : $73,1.968$

21. KLAUS, M. Reiss, O.K., Tooley, W.H. y col.: Science 137:750, 1.962

22. MONTOJAMA, E.K., Kikkawa, Y, Orzalesi, M. y col. Pediatrics 48:547, 1.971

23. KO'TAS, R.V.: J. Pediat, 81:378, 1.972

24. MONTSERRAT de M. Fench, PHD, and Dan Tulchinsky, M.D. Total Cortisol in Amniotic Fluid and fetal lung maduration. The New England Journal of Medicine. January 16:3, 1975

25. PATTLE, R.E.: Nature 175:1125, 1.955

26. REID, L.: Ciba Foundation Symposium. Lit tle, Brown and Co. 1.967

27, ROUX, J.F. Nokamura, J., Brown, E. y col. : Pediatrics, 49:464, 1.972

28. STRANG, L.B.: Little, Brown, and Co., 1967

29. STRANG, L.B, Anderson, G.S. y Platt, J.W.: $1: 954,1.957$

30. SC ARPELLI, E.M.: Triangle, 10:47 1.971 